\title{
An Insight into the Difficulties of Software Development Projects in the Pharmaceutical Industry
}

\author{
A. Hajou, R. S. Batenburg, and S. Jansen
}

\begin{abstract}
Software development projects in the pharmaceutical industry are highly regulated as software failures can have a direct impact on the quality of medicinal product and therefore patient safety. Regulation is imposed from a governmental level but is also employed in the form of comprehensive industry standards. The strive for regulatory compliancy is a direct cause for high overhead in software development projects, which leads to the procrastination of innovation. 14 interviews have been conducted with domain experts in a quest to understand and validate the difficulties of software development projects in the context of the pharmaceutical industry. The interviews focussed on the difficulties of software development projects in the pharmaceutical context and the lack of adoption of agile methods, as these have proven to reduce overhead of software development projects in other industries. The collected information is disclosed for the purpose of advancing the research area which is characterised to be minimally researched.
\end{abstract}

$\begin{array}{ccrl}\text { Index } & \text { Terms-Agile } & \text { pharmaceutical } & \begin{array}{r}\text { industry, } \\ \text { problem-centered interviewing, regulatory }\end{array}\end{array}$ software development.

\section{INTRODUCTION}

Software development is subjected to a constant pace of maturation. This development is driven by practical and scientific effort to improve software development projects. A fast changing field of research is agile software development. Agile has been more and more the subject of scientific studies [1]. Agile has received many definitions in the last decade but its core purpose is reflected best in the original definition: "We are uncovering better ways of developing software by doing it and helping others do it. Through this work we have come to value:

- Individuals and interactions over processes and tools

- Working software over comprehensive documentation

- Customer collaboration over contract negotiation

- Responding to change over following a plan" [2].

Where methods that are considered to be 'agile' (e.g. Scrum, XP) are researched, applied and evaluated often in many industries, they lack the suitability with regulated environments (e.g. the pharmaceutical industry). McHugh et al. mention that "for agile methods to succeed, an organisation must be structured in a way to accommodate

Manuscript received June 21, 2014; revised August 29, 2014. The work described was sponsored by Magna Medical B. V. in Nijmegen, the Netherlands. We are grateful for the generous input and constructive guidance.

The authors are with the Department of Information and Computer Science at Utrecht University, the Netherlands (e-mail: a.hajou@students.uu.nl,r.s.batenburg@uu.nl, s.jansen@uu.nl). agile methods" [3]. McHugh, Cawley, McCaffery, Richardson and Wang summarize that there is evidence of gaining significant organisational benefits upon the adoption of an agile method for managing and performing software development projects [4]. While there is sufficient of scientific literature on organisational requirements for optimal agile adoption, there is no literature elaborating this knowledge about pharmaceutical companies. Fitzgerald, Stol, O'Sullivan and O'Brian mention the lack of research is due to the fact that "Agile methods and regulated environments are often seen as fundamentally incompatible" [5], while Heeager and Nielsen mention that "there are few reports on this issue [incompatibility between agile and document-driven development processes] and even less empirical research documenting the assumed incompatibility" [6]. Both of the statements have little scientific backing, as the number of literature related to agile adoption in the context of the pharmaceutical industry is minimal. Evident is the impact caused by the obligation (i.e. for pharmaceutical companies conducting software development projects) to adhere to regulatory and industry standards, such as EU GMP Annex 11 and ISPE's GAMP5 [7], [8]. Unclear is what causes the described incompatibility.

Therefore, as a part of a more extensive research [9], an attempt has been done to gain understanding of the field of software development methods in the formal context of the pharmaceutical industry. Special emphasis has been given to the use agile methods and the difficulties of conducting software development projects in this particular industry.

The purpose of disclosing the collected information is to support the advancement of scientific research in this specific research area.

\section{RESEARCH APPROACH}

Previous research identified a total of 3 holistic problem categories (PC) that describe the difficulties of software development projects in the context of the pharmaceutical industry [10]. The PCs have been denoted as 1; "the regulatory complexity of software development in the pharmaceutical industry", 2; the "differences between the agile and the highly documentative approaches", and 3; the "lack of attempts to be agile in the pharmaceutical industry" [10]. The PCs describe multiple problems that were identified from scientific literature. Unfortunately, due to the lack of specific literature about software development projects in the pharmaceutical industry, related fields of research (e.g. medical device, safety critical software) have been researched for the identification of problems occurring in software development projects. The identified problems required additional validation on whether these were 
applicable in the context of the pharmaceutical industry. Therefore these 3 PCs have been split up in 14 isolated problem subjects (i.e. abbreviated to PS, extracted from the $\mathrm{PC}$ descriptions) to be used during expert interviews.

\section{A. PC1 Consisted of the PSs}

- "Multi-layered regulatory requirements" (i.e. the complexity induced by the high level of overlapping regulatory project and product requirements);

- "Large initial investment for third parties" (i.e. the high threshold for third parties to introduce new products or practices in and for the pharmaceutical industry);

- "Fast changing regulation and legislation";

- "Old fashioned attitude" (i.e. old-fashioned attitude of manual and rigorous project governance).

\section{B. PC2 Was Split up on the PSs}

- "Preference of defined logic over empirical logic" (i.e. the focus on a strict predefined process instead of focusing on graduate procedural improvements);

- "Requirements traceability" (i.e. the traceability of software requirement evolution);

- "Documentative attitude" (i.e. the comprehensive habit of documentation generation during IT projects);

- "Estimation difficulties" (i.e. the difficulties and possibly the illusion of prospective planning);

- "Risk oriented decision making" (i.e. a malfunctioning risk oriented discourse within IT projects).

\section{PC3 Was Split up in the PSs}

- "Minimal research" (i.e. the lack of scientific research of the use of agile methods in the context of the pharmaceutical industry);

- "The lack of embracing change" (i.e. the conservative attitude of the pharmaceutical industry which the complete opposite of agile's pursue to change);

- "Absence of project evaluation" (i.e. the absence of post-project evaluations for improving a procedure);

- "Obligation of traceable compliancy" (i.e. requirement of tracing processes, decisions and their effect on project deliverables);

- "Palmed obstruction" (i.e. refraining procedural change due to the statement that the problems are 'industry wide'). Expert interviews have been conducted using Witzel's Problem-Centered Interviewing method [11]. PCI allows for custom tailoring questions (i.e. based on the PSs) to generate an informal environment in which the respondent can tell his/her story instead of engaging in a question-answer process. A positive effect of PCI is that there is more room to elaborate on PSs that are perceived to be relevant and negatively influencing their day to day operations. A negative effect is that the PSs that are not perceived as an issue that matters are often omitted.

The contacted experts were required to be tightly involved with IT projects (e.g. software development, software implementation), had to have experience with software validation, and had to be operating in the Dutch pharmaceutical industry (i.e. due to logistical limitations). Observed is the limited number of persons operating in this specific niche. A total of 55 persons have been contacted which lead to 14 interviews. 10 were held face-to-face, 3 were held via the telephone and 1 was conducted via email.
The experts ranged from managerial to operational level, and operated in companies that ranged from large multinational pharmaceutical enterprises to small subcontracted consultancy firms. The interviews have been fully transcribed to be used for problem statement extraction (i.e. expert's reaction towards the PSs). The extraction process has been conducted using the software product 'NVivo 10' from QSR international in which the problem statements were selected and connected to nodes representing PSs.

The extraction of problem statements related to the PS resulted in a list of 317 combinations to be used to verify the 14 PSs' validity.

Problem statements could either acknowledge a PS as an actual problem, or disagree with a PS. Every PS will receive an acknowledgement-percentage-score determining the validity of a PS as a problem that has been experienced in practice.

\section{RESUlTS}

\section{A. Problem Subject Popularity}

The interviews varied in discussed subjects and level of detail. Every single PS has been a subject of discussion at least 5 times throughout the interviews. This means that not all PS have been discussed in every interview. Fig. 1 shows the number of extracted citations related to the PS, indicating the PS popularity. The PS 'Documentative attitude' has been discussed the most as it has been 60 times subject of discussion during the 14 interviews, whereas the topic 'Estimation difficulties' is only discussed 5 times.

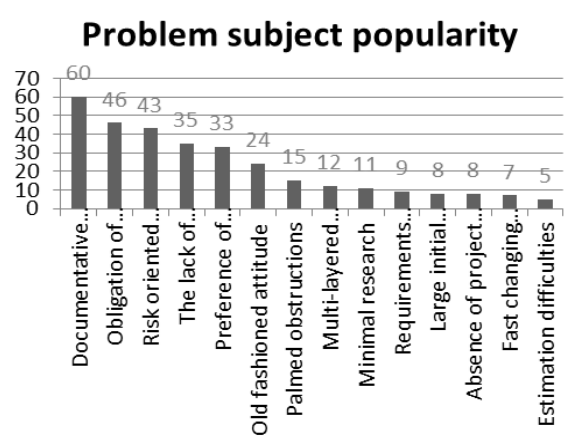

Fig. 1. Number of citations per PS indicating subject popularity.

PSs that can be considered as generic project difficulties (e.g. leaving out project evaluations, adaptability to project structure changes, planning and estimation difficulties) have been discussed far less than PSs that identify IT projects in the context of the pharmaceutical industry (e.g. documentative, compliancy driven, risk oriented decision making).

\section{B. The Experts' Perspective on the Problem Statements}

The 14 PSs have been positioned in this chapter based on the PS popularity as illustrated in Fig. 1. Due to article length limitations, a representative subset of the 317 problem statements are provided per PS. To add context into the citations (i.e. to enhance understandability), additional supportive sentences are added between brackets.

\section{1) Documentative attitude}

The PS describing the documentative nature of practices within the pharmaceutical industry has been discussed the 
most. From the 60 statements that were extracted from the transcriptions, $55(91.7 \%)$ acknowledge the problem subject as an actual problem. The problem is so peculiar that one expert made a picture of a stack of test documentation that the expert's team created and tried to show it during the interview:

"And I would like to show you a picture of that, because it eventually became a pile 'this' high with documents that we had to deliver" (interview 11).

The documentative culture is primarily caused by the need for compliancy to regulations and the fear for regulatory inspections. The current industry standards mainly fuel the need for overhead due to the fear of 'missing information':

"There are quite some people using text books in which they acknowledge and use every single part, and do not attempt to find any room for adaptation. They will do the $150 \%$. That's how you become slow and rigid" (interview 5).

The reasoning behind the creation of excessive documentation (i.e. omitting risk of incompliancy) is completely counteracted by the deed itself. The more over-complete the documentation is, the more risk there is for missing links or inconsistencies:

"It introduces risk when you create a lot of documentation. You are trying to cover all sections by creating excessive documents, resulting in the situation that more and more inconsistencies between documents are created. So, which of the documents would then be the truth?" (interview 12).

Thick documents do not necessarily have to be in the favour of the project team. The inconsistencies between documents can raise questions, but that is only if an inspector does not trust the situation and reviews the documents in detail:

"An inspector must have a reason to not trust a situation. Then, a thick document is against you. Otherwise, I think, a thick document is in your favour" (interview 14).

Then, there is the problem of reviewing the documents, which often is done by persons that were not involved in the creation of it. Due to the lack of knowledge (i.e. details and backgrounds that resulted in the documented sections) reviewing can become a long and slow process:

"The more they evaluate, the more they complicate" (interview 9).

It is clear that the interpretation of how and how much documentation should be created differs due to conflicting interests. Inspectors would like to see strict processes that enhance quality which is traceable on paper, while project team members are kept in the delirium by constantly hearing that they need to create 'enough' documentation:

"We once asked ourselves: 'We are software developers, how do we know that we generate enough documentation?" (interview 3).

"And they said: 'Well, that document is quite small. This will probably not end well. It is just 4 sides... You should give the document a bit more body, right?"' (interview 14).

"We will have to document it, because if it ain't written, it ain't exist. And that is quite important" (interview 2).
"Creating documents, well, that has been the eternal discussion. You should not write documents just to fill paper. It should have a purpose. And if you do not now the purpose, you probably don't know what to write" (interview 3).

2) Obligation of traceable compliancy

The PS that identifies the need for tracing the process for accomplishing regulatory compliancy has been discussed in a more diverse manner. There is no clear consensus on whether the practices related to the traceability of compliancy is considered a problem. The statements who disagree that this problem subject is actually a problem has the majority, but only with a small margin (24 out of $46,52.2 \%$ ). This is mainly due to the fact that it is a clear and mandatory regulation:

"Traceability is a regulatory requirement" (interview 3).

"They said: 'if there is no traceability matrix, we consider the system not validated. And I fully agree to that" (interview 11).

The general acceptation of this procedure is related to the quality driven nature of the pharmaceutical industry:

"At least they can never mention that I have not been concerned about quality" (interview 12).

"We are eventually accountable for everything, but that is how it is. The GAMP clearly says that the regulated company is responsible for the validated state of the complete system. That requires assessment of the quality system of the supplier, the integrator, by performing a supplier audit. We then check whether they use a bible like the one we use. We chose GAMP because the industry that we are in generally accepted it too" (interview 1).

The problem is: how far should one go in indulging practices to register the process and its compliancy steps:

"You could use a calculator to calculate it [Excel formulas]. And sometimes that was possible, and sometimes not. But the quality officer went a step further, mentioning; is the calculator you are using validated?" (interview 11).

"You then are reviewing a document with 10 persons, word by word. Where one has a word there and the other has something else here..." (interview 10).

"What I said earlier, the industry is making itself crazy by employing large quantities of IT and QA [i.e. Quality Assurance] personnel to just focus on that extra 20\% [of work that requires $80 \%$ of the time]. Those difficultly interpretable 20\%" (interview 2).

In essence, the extra work to register the process to allow traceability in decisions and plan deviations is done solely for the purpose of creating a trail of evidence:

"You will have to prove it to the outer world. If you receive an inspection, you need to prove that you are in control", (interview 11).

\section{3) Risk oriented decision making}

Observed is that there is general consensus (29 out of 43, $67.4 \%$ ) about to the notion that the risk oriented practices are not perceived as a problem during IT projects. However, the practice of risk oriented decision making has proven to be difficult: 
"If everyone does the same thing [executing project steps using a checklist], it might be very effective, but not very efficient. So there are opportunities there. The described risk based approach tries to enforce that to a large extent, pragmatically. But people have to consider and identify potential risks. And people tend to find that difficult" (interview 3).

"And if you say something like; 'okay people, we did an impact analysis and we choose to not validate it'. People will respond like; 'wow, wait a second. We are in a pharmaceutical business here, you cannot just put that thing down and use it?'. They [QA representatives] will directly search for arguments that can have great impact" (interview 6).

The fact that risk oriented operations prove to be difficult results in a risk avoiding mentality instead of a risk oriented mentality:

"The fear reigns. So let's not touch it and take zero risk" (interview 5).

"The amount of extra work that it [software update] introduces... Potentially related to some unfamiliarity towards validation... It results in risk avoiding situations, where there is no validation because we remain the old situation" (interview 4).

Balancing between incompliant but fast software development and compliant but slow and rigorous software development seems to be highly influenced by fear:

"It often is related to whether the company has been provided a slap on the wrist. You would then see that everyone switches over to just doing everything [creating all possible documents and performing all described processes], and not taking any risk whatsoever. Working down the complete checklist. After a few years, the fear reduces and projects are executed more smoothly by taking and calculating more risk into the project. It is pretty much related to which company you are in and its [recent audit] history" (interview 8).

"Otherwise you have the risk that there is a critical requirement that has been forgotten to be included in the tests. And due to taking the risk of omitting certain tests, it can happen that eventually something just is not working" (interview 8).

Using the risk factor in the consideration to perform steps is generally accepted but lacks good practical implementation. An option would be to advice a general method that is easy to understand and delimited for fast execution:

"I would really like to see a standardized risk analysis method for validation of different types of systems" (interview 8).

\section{4) The lack of embracing change}

Agile propagates that project team members should embrace change for fast adaptability to problems and quick delivery of solutions. Expecting procedural or requirement changes initiates another mentality than following a planned approach. This mentality seems to be missing in software development projects in the pharmaceutical industry. And therefore 20 out of 35 statements $(57.1 \%)$ acknowledge that missing this potentially essential attribute is causing problems. Whereas 15 of the 35 statements $(42.9 \%)$ do not perceive this problem. The absence of this attribute is inherited from the regulatory pressure. IT team has to endure these difficulties:

"Sometimes you would really like to propose and work on improvements. But the ballast of regulations and legislation prevents you to do so. You already see what you have to do if you choose to improve. Documentation, alteration or work instructions et cetera" (interview 7).

"So after 2 or 3 years of no improvements you enter the stage in which there are systems that are validated but horribly out-dated. Eventually, the support of the supplier will be stopped and therefore, in itself, people are working towards incompliancy. They [project stakeholders] do not even recognize that" (interview 5).

Furthermore, obstructing potentially uncontrolled change is the sole purpose of existence for some people. And if the controls have a different pace than the rigorous processes of these stakeholders, they will have difficulties accepting it:

"So you would think; 'why are projects not use another method, another tactic, which led to positive results at many other companies'. In that case, I fully agree that agile can be a pretty good method, especially for the business. To remain within budget, deliver good products that satisfy customer needs. All of that would be due to agile. But there will always be the march to the Quality Assurance department that will express their difficulties with the method, because you are going to do something different" (interview 6).

"They [software development department] are international teams of thousands of people that have a very strong lobby towards agile. They mention that it is the future, that it is ready and that it requires approval. QA people do not like that at all. They are very cocky in that sense" (interview 14).

The notion of embracing change could be implemented within the boundaries of the project processes as we know them. Current practice is to review product evolution by reviewing documents (e.g. functional specification, design specification). By reviewing prototypes instead of documents direct feedback can be provided by the end-user, instead of providing feedback on the functional specification and eventually also on the prototype:

"The principle in which agile can play a major role is reviewing based on risk and a prototype. Why would one require to first review an old-fashioned document? It should be possible to review a prototype instead?" (interview 3 ).

\section{5) Preference of defined logic over empirical logic}

The PS that opened discussions regarding to the aspiration of defined logic over an adaptive and more empirical logic has not received exhaustive consensus. 19 out of 33 statements $(57.6 \%)$ acknowledge that this is a problem, while 14 statements $(42.4 \%)$ do not. Defined processes, such as processes dictated by industry standards, are generally adopted one-to-one. Instead of adapting methodologies to the company, people tend to baptize it into the new golden standard:

"Those people [QA staff] hide behind the regulation. Maybe it is the type of person, but they do not face a 
discussion with authorities. I think that it should be part of what you do" (interview 5).

But adapting a method prior adoption requires energy, knowledge and commitment:

"You will need to have experience in applying the rules in practice. Only then, you are an expert in the regulation" (interview 14).

"But, is validation than that complicated? No. We just want to go out of the way of additional work. This just generates more work" (interview 4).

By not adapting methods, based on experience and company culture, large projects can go wrong as these rigorous unadapted methods conflict with each other:

"What I find very important, and what often fails in many projects, especially in IT, is the integration. The integration of multiple stakeholders from multiple organisations. They often have their own methods of validation" (interview 6).

"You can foresee that these things fail and a million euro project is about to get ruined because they [adopted methodologies at various parts of the company] do not match" (interview 6).

The mixed statements do not clearly identify the pharmaceutical industry's or the IT project-stakeholders' potentially dogmatic practices. Technology that is clearly proven lowers the fear that comes with new products, and is therefore adopted faster:

"I think that it will help to use COTS [Commercial of the Shelf] software. It makes it easier. If we use something like SAP, a COTS product, and were are going to use COTS templates with it that have been already proven... It will definitely mean that the number of software faults is minimal, or zero, and that the number of process or implementation errors is much smaller" (interview 4).

"I do not see any reason why it [an agile method] cannot work in pharma. A reason might be the unfamiliarity. A reason might be fear, or that it is not 'proven technology'. I do not know" (interview 12).

The industry is moving on from dogmatic and therefore defined practices. Traditionally regulation or guidelines were interpreted almost literally. They now are interpreted on a more practical level:

"When 21 CFR Part 11 was introduced, or at least, the whole regulation around GAMP4, people were fairly rigid. At least, that is how I saw it. 'This is how it should be done', or whatever. But it restricted innovation throughout the industry, which resulted in a more practical attitude towards GAMP5" (interview 11).

\section{6) Old fashioned attitude}

Most of the statements from the experts agree on the old fashioned mentality of the pharmaceutical industry. 20 out of the 24 statements $(83.3 \%)$ on this PS acknowledge it to be a problem. Although, many experts mention the cause of this problem to be the type of stakeholders:

"If you look to the people, you can see that there are people that are carved out of oak. These validation specialists have their job to align our practices with GMP regulations. They will do anything, because eventually that's how it should be, to make sure we do the things [rigorous validation] we should do" (interview 4).

"Those departments [quality assurance] are often filled with... I do not want to say 'old men' but eventually that is what they are. They often are people that made a career in the pharmaceutical industry and that are promoted due to their process knowledge to the quality department" (interview 6).

It does not necessarily have to be the persons that are the cause of the 'old fashioned' way of thinking, it often is the companies' culture:

"It is not always the compliance that is problematic. It often is the culture in this corporate environment. Inside corporate environments you often see people working there for a very long time. This induces a culture of 'this is not how we do these things" (interview 5).

"On the moment that I executed the tests, it had to be done by hand. All questions had to be answered one for one. With a paraph and so forth. Eventually, the higher management accepted the test results to be created in Word" (interview 11).

It clearly is an after effect of old practices which every now and then reoccur in current projects:

"I have always aspired to add validation steps throughout the automation process. Back in the days people thought 'we automate and then validate. Just because we have to'. But the validation part did not add anything to the automation part except for rigorous testing. But you can accomplish that without validation. It could be the case that everything was tested very well, but there was no time left for validation. That is also how it went" (interview 3).

"If you just have to validate $30 \%$ of it, back in the days, in the $O Q$ [operational qualification], you had to validate everything in the same way" (interview 3 ).

According to the statements there is a sense that the practices have evolved, but have also inherited out-dated reasoning.

7) Palmed obstruction

Not only the interviewed experts face the problems that have been identified as PSs in this research. 13 out of 15 statements $(86.7 \%)$ refer to other stakeholders who suffer from the same problems. The ability to cope with the stated problem statements differs between the type of job one has. IT often lacks detailed knowledge about the pharmaceutical industry, while the pharmaceutical industry often lacks detailed knowledge about IT:

"I have been an IT manager of a big IT department. That is of a pharmaceutical IT department. They [IT staff] should do more to understand something of compliance, because they don't', (interview 5).

"Everyone knows that we are required to validate. Maybe that personnel at the lowest level is not involved or does not have any interest in that, but everyone on a certain level knows that [validation obligation]. It is part of the job" (interview 11).

"What I often hear is that the authorities that perform the inspections have no clue on how software is constructed. 
They fail in performing proper inspections, so to say" (interview 6).

But apparently the problems are not omitted because some personnel benefit from them:

"Validation and rigorous QA often is their right to exist. Maintaining the problem and therefore the complexity might be done consciously. Therefore I remain to the point that validation should be demythologised. And the knowledge about validation should be spread out more" (interview 4).

\section{8) Multi-layered regulatory requirements}

The many regulatory requirements related to IT products and processes in the pharmaceutical industry provide for a high level of complexity. 9 out of 12 statements (75\%) agree that this PS is considered to be a real and existing problem. However, due to the close relation between the practices of the pharmaceutical industry and the safety of patients, it is generally accepted that this high level of complexity is 'just part of the job':

"It is not only IT, but essentially everything you do in pharma, whenever something is changed or new devices that are not related to IT are implemented... Everything has to be done according to rules and procedures. You cannot just change something in a system. There is a very specific change process that is involved. It sure puts a lot of pressure on the organisation to accomplish this, which is quite different at other types of companies" (interview 6).

"It [quality requirements] is nothing new. It is just very specific and very detailed" (interview 14).

"On the other hand, you might say; ' a country differs from another country. Local laws are different, but unfortunately there are not many of those'. Most legislation is related to the EU [European Union] or FDA [Food and Drug Administration]" (interview 1).

\section{9) Minimal research}

Only 1 of the 11 statements $(9.1 \%)$ related to the PS describing the minimal investigatory effort in software development in the pharmaceutical industry does not recognise this as a problem. An almost unanimous opinion conclude that software development project suffer from difficulties due to the closed attitude of the pharmaceutical industry:

"A lot is 'kept within the own organisation'. The high level of regulation and legislation force everyone to struggle with the operations, while sharing or inspiring the outside world could help" (interview 7).

"If I look to my colleagues, of which I have about 70 in our team, only 2 or 3 are a little bit familiar with the pharmaceutical industry. Nobody else" (interview 11).

Influences from the outside are systematically blocked, unless they are induced from inside the industry:

"There is a technology push going on in a sense that 'this is how we are going to work'. Everyone will be in shock, mentioning things like 'no, it is not compliant, this is not how we do things'. It almost is a natural reaction. Agile originates from IT, while GAMP originates from quality" (interview 14).

On the other hand, there is limited interest in the practices of the pharmaceutical industry:

"Pharmaceuticals is not a 'trendy' sector. It is old and dusty, and all those regulations... Well, who is making pills? There is no fun in doing it. Not really. But it is good money" (interview 1).

\section{0) Requirements traceability}

Requirements traceability is a regulatory obligation. This might be the cause that most of the statements related to this subject, 6 out of $9(66.7 \%)$, do not acknowledge that this is a problem. It is a discipline that allows the project team to demonstrate evidence of validation. It just is extra work:

"What you often see is that you are asked to create a requirements traceability matrix that covers all requirements and their tests. If they are not tested, it should be documented why it is not tested and that it cannot be a cause of problems. Eventually there should be some kind of report that mentions that all is good. All of these extras is additional work though" (interview 8).

"Traceability is a regulatory obligation. Somehow your requirements should be traceable to, but this is old-fashioned; a document such as a functional specification, a detailed specification and a test script. If the test script is traceable, intermediate steps can be omitted. These intermediate steps are just there to ensure the test not to fail" (interview 3).

The traceability of requirements often is seen as an essential part of an IT project, allowing high level management of the process:

"The traceability of a requirement is done from specification until the testing and until its final verdict. Requirements are also subject to versioning. And what you say is completely true; it is common practice. Requirements change because you become wiser. You know more and therefore they change. This change is translated and used throughout the process. What is difficult is that all of these requirements have to be managed. And the list can be huge in cases of large projects such as SAP. The challenge is to keep it [requirements traceability matrix] in line with practices and update and document it as fast as possible" (interview 1).

\section{1) Large initial investment for third parties}

The limited inflow of external parties in the pharmaceutical industry is very limited due to the large initial investment in getting compliancy, knowledge and the chance to deliver. 7 out of $8(87.5 \%)$ of the statements acknowledge this as an existing problem. The step is too big and the market is too small:

"It is a small market that has high demands and a difficult threshold of entrance. You will need to have a lot of detailed knowledge about the area [software development in the context of the pharmaceutical industry], which you will not receive on beforehand. So therefore it is a difficult niche to enter. And, as you just said, it is practically only people that already worked in the industry to be able to start something new [new product or new company]" (interview 4).

"So what you see, and I have a similar feeling about that, is that the threshold becomes higher and higher. So I question myself on whether this ends or not. Because eventually, and I kid you not, the cost aspect is enormous" (interview 2). 
But after establishment in the pharmaceutical industry, one is able to commercially expand itself greatly:

"But then again, what you often see is that if you are inside one of those companies [pharmaceutical companies], you have lots to sell" (interview 8).

Therefore one can perceive that the initial difficulties can pay off when a commercial third party is able to push through the regulatory boundaries.

\section{2) Absence of project evaluation}

Project evaluation is a generic problem that occurs in different types of projects. It is not different in the pharmaceutical industry as it has not been discussed often, and there is an equal opinion on whether it is a problem or not. 4 out of 8 statements $(50 \%)$ perceive it as a problem.

"It always starts with writing down the requirements, which is something people find very difficult. If you eventually evaluate them, you see the same thing. They are not well formulated, not clear and very much scrambled. 3 requirements are stuffed into 1 , making 1 of them critical and 2 not. The whole requirement will become critical that way. If you would split those, only a third would be critical. People find that very difficult. People did not learn how to do this, and I try to train them in that. How difficult it may be. Evaluation should also be done during the project" (interview 3).

"But in this case we have a phase of evaluation which we actually really postpone. Then we postpone it again" (interview 9).

The difficulties resulting from the absence of project evaluations therefore are not perceived as being 'typical' for the pharmaceutical industry.

\section{3) Fast changing regulation and legislation}

The PS about fast changing regulatory requirements is acknowledged as an actual problem. 6 out of 7 statements $(85,7 \%)$ related to this problem statement find it difficult to cope with the fast changing regulatory requirements. However, the low number of statements illustrates that the subject has not been discussed often. Most statements mention the novelty of IT in the pharmaceutical industry, but do not focus on the changes in regulations:

"What is also the case is that it [software and software development] is $100 \%$ new for the pharmaceutical industry. IT never was very important" (interview 6).

The response on changes, whether they originate from regulations or from IT, is rather rigorous and lacks the pace that is needed to properly adapt:

"So if you find out that regulations are changing, or that the needs are changing, you can initiate a change. The change will be discussed in the advisory board in which there will be discussed whether we are going to do it, what is the impact, the costs, the benefits and the effect of not implementing it" (interview 8).

The responses unfortunately were highly generic and did not focus on the difficulties of complying to fast changing regulatory requirements.

\section{4) Estimation difficulties}

The discussed difficulties regarding to project planning and estimation were quite generic, and not so much specific for the pharmaceutical industry. From the 5 statements 4 $(80 \%)$ acknowledge it as a problem while 1 statement does not. The statement that does not acknowledge it as a problem acknowledges is as a necessary evil in IT projects:

"It mostly is experience that you use to estimate how long it will take to write a document, to know how long it takes to work through the process and approvals. All these variables are taken into account when creating an estimation. It mostly is gut-feeling. If an application increases in size, then the documentation will become a bit more elaborate. It takes some more time but you can oversee that quite well" (interview 8).

However, the rigorous waterfall method-related approach that is employed also making project estimations very difficult. Budget determination is often done before requirements collection. How can one determine a budget when there is no clearance on what to be built?

"What often happens is that you will have to mention the prospected budget that is required to accomplish the project. But only after receiving the 'go', you are going to collect and write down the requirements. How can you predict the correct budget or planning this way?" (interview 8).

The rather trivial statements support the notion that the generic project problems lacked elaboration during the interviews.

\section{CONCLUSION}

The lack of scientific research done in the area of software development methods in the context of the pharmaceutical industry is reflected in the lack of related scientific papers. In order to scientific research in this area 14 interviews have been conducted with experts in the field of IT projects in the formal context of the pharmaceutical industry. 317 problem statements have been extracted which either acknowledge or disagree with previously stated problem subjects.

The experts were almost mutually conclusive about the project impediments that are caused by the documentative habit of conducting software development projects. The lack of scientific research, adoption of new methodologies and intervention of third party companies ensures that the pharmaceutical industry does not evolve in term of software development practices. Where agile software development methods are gaining adoption across industries, the pharmaceutical industry is lagging behind.

There is a strong opinion among the experts on the role of quality assuring departments in software development projects. Their collaboration is often perceived as necessary but restrictive.

The importance and evolving speed of IT in general is acknowledged by the field, but as it comes with a different etiquette, it requires mutual adaptation.

\section{DISCUSSION AND FURTHER RESEARCH}

The novelty of the research area introduces several factors impacting the overall reliability of the research. The initial 
drawback is the 14 identified problem subjects, as these were based on literature related but not specifically defined for the pharmaceutical industry. However, the validation of the PS by discussing these with 14 domain experts increases the overall reliability. Broader research, involving more domain experts can define a more holistic conclusion per PS which delimits the area in which solutions can be applied.

Problem-Centered Interviewing allows for receiving much valuable 'inside' information, but is often limited to a few discussion topics. Other problems might retain the informal atmosphere of interviewing but allow for clear and defined interview topics. However, the fact that minimal effort has been done by the experts to discuss problems that appear to be regularly present in software development methods, identifies the potentially unimportance of them.

Finally, the article length restrictions forced to select citations (i.e. problem statements) that were representative for the complete set of PS related statements. This selection is highly subjective as it is based on the interpretation of the citation and its textual context.

\section{REFERENCES}

[1] T. Dings $\varnothing y r$, S. Nerur, V. Balijepally, and N. Moe, "A decade of agile methodologies: Towards explaining agile software development," The Journal of Systems and Software, vol. 85, no. 6, pp. 1213-1221, 2012.

[2] K. Beck, M. Beedle, A. V. Bennekum et al. (January 2014). Manifesto for agile software development. agilemanifesto.org. [Online]. Available: http://agilemanifesto.org/

[3] M. McHugh, F. McCaffery, B. Fitzgerald, K. Stol, V. Casey, and G. Coady, "Balancing agility and discipline in a medical device software organisation," Software Process Improvement and Capability Determination, pp. 199-210, 2013.

[4] M. McHugh, O. Cawley, F. McCaffery, I. Richardson, and X. Wang, "An agile v-model for medical device software development to overcome the challenges with plan-driven software development lifecycles," in Proc. 5th International Workshop on Software Engineering in Health Care, 2013, pp. 12-19.
[5] B. Fitzgerald, K. Stol, R. O'Sullivan, and D. O'Brien, "Scaling agile methods to regulated environments: An industry case study," in Proc. the 2013 International Conference on Software Engineering, pp. 863-872, San Francisco: IEEE Press, 2013.

[6] L. Heeager and P. Nielsen, "Agile software development and its compatibility with a document-driven approach? A case study," in Proc. 20th Australasian Conference on Information Systems, pp. 205-214, Melbourne, 2009.

[7] EudraLex - Volume 4 GMP Annex 11: Computerized Systems, European Commission - 2011

[8] GAMP5, A Risk-Based Approach to Compliant GxP Computerized Systems, ISPE, Tampa, FL, 2008.

[9] A. Hajou, "Shaping agile software development methods for the highly documentative pharmaceutical industry," M.S. thesis, Dept. Inf. CS., Utrecht University, Utrecht, the Netherlands, 2014.

[10] A. Hajou, R. S. Batenburg, and S. Jansen, "How the pharmaceutical industry and agile software development methods conflict," in Proc. 14th International Conference on Computational Science and Its Applications, Guimarães, Portugal: IEEE, 2014, pp. 40-48.

[11] A. Witzel, "The problem-centered interview," Forum: Qualitative Social Research, vol. 1, no. 1, 2000.

Ali Hajou was born in 1987. He is a graduate student at the master study in business informatics at Utrecht University in the Netherlands. His research interests include software development practices in high governance environments. $\mathrm{He}$ is focused on the applicability of agile practices in the domain of software development in the pharmaceutical industry

Ronald Batenburg was born in 1964. He obtained his masters in sociology at Utrecht University and his Ph.D. in social sciences at Groningen University. Since then, he worked at the Social and Cultural Planning Office as a research fellow, at Utrecht University as post-doc researcher, at Tilburg University, and Nijmegen University as an assistant professor, as an associate professor at Utrecht University and as senior research consultant at Dialogic Innovation and Interaction. From April 2009, Dr. Batenburg is a program coordinator at health care and manpower planning at NIVEL, the Netherlands Institute for Health Services Research. His research interests are labour market studies and transitions, as well as the socio-organizational aspects of ICT.

Slinger Jansen was born in 1980. He is an assistant professor at the Department of Information and Computer Science at Utrecht University. He is one of the leading researchers in the domain of software ecosystems and co-founders of the International Conference on Software Business and International Workshop on Software Ecosystems. He is the lead editor of the book Software Ecosystems: Analyzing and Managing Business Networks in the Software Industry and of several others. 